\title{
Cuestionario sobre adaptaciones de acceso para directores de instituciones educativas inclusivas.
}

Dra. Esperanza Bernaola Lic. Gladys Quintana Lecaros

\section{Resumen}

Estudio para determinar las características psicométricas del cuestionario sobre adaptaciones de acceso para directores de instituciones educativas inclusivas de la RED 02 de la UGEL 06 de Lima Metropolitana. Se trata de un estudio descriptivo instrumental llevado a cabo en una muestra de directivos de 14 instituciones. Se construyó un cuestionario con 28 ítems, pero luego del análisis de los ítems quedó conformado por 18 ítems que abarcan tres ámbitos: Contexto local, Centro escolar y Contexto Aula. Se concluye que las escalas del Cuestionario sobre Adaptaciones de Acceso para Directores de las IEI tienen una fiabilidad aceptable: Escala Contexto local $(0,695)$, Centro escolar $(0,734)$, Contexto aula $(0,787)$, Escala Total $(0,701)$. La validez se estableció a través del análisis de su estructura interna empleando la técnica de la intercorrelación de las escalas del instrumento. Empíricamente las escalas contexto local, centro escolar y contexto aula miden aspectos bastante independientes entre sí, encontrándose reflejada en la puntuación total con mayor peso Centro escolar y Contexto aula, y en menor peso Contexto local. En suma, la apreciación de la información del análisis de los ítems, de los coeficientes alfa y de la intercorrelación de las escalas del Cuestionario de Acceso a las IEI, permite decir que en la muestra analizada el cuestionario tiene un comportamiento psicométrico de características básicas que lo hace útil para cumplir con el propósito para el que fue construido.

Palabras claves: Adaptaciones de acceso, validez, confiabilidad Contexto local, Centro escolar y contexto aula. 


\section{Introducción}

Unos de los aspectos a desarrollar en una escuela inclusiva es el hacer de las Instituciones espacios educativos accesibles a los estudiantes y a cada una de las personas de la comunidad educativa (docentes, directivos, padres de familia, personal administrativo, visitantes, etc.). Es decir, que cada una de las personas que acudan a la escuela tengan la misma oportunidad de utilizar de manera autónoma, todos y cada uno de los servicios que se ofertan en ella independientemente de las capacidades físicas, psíquicas, cognitivas, sensoriales, económicas o culturales.

En el año 2006, en Nueva York se llevó a cabo la Convención sobre los Derechos de las Personas con Discapacidad organizada por las Naciones Unidas se enfatizó que, con respecto a la accesibilidad, los países deben identificar y eliminar los obstáculos y barreras de acceso de modo que las personas con discapacidad puedan tener para acceder, en igualdad de oportunidades, a su entorno físico, al transporte, las instalaciones y los servicios públicos, y las tecnologías de la información y las comunicaciones (artículo 9).

Los Estados deben asegurar que las personas con discapacidad puedan acceder a la educación primaria y secundaria, la formación profesional, la enseñanza de adultos y el aprendizaje permanente sin discriminación y en igualdad de condiciones que los demás (Aragall, 2010).

En Perú se priorizó la capacitación y elaboración de materiales como "guía para las adaptaciones curriculares", postergándose el desarrollo de aspectos de accesibilidad a elementos físicos como la arquitectura, mobiliario y su organización en el centro y aula.

En nuestro país, el Ministerio de Educación, consecuente con la Educación Inclusiva que propugna, se encuentra desde el 2013 realizando acciones en correspondencia al Programa Presupuestal por Resultados 0106 en la que el Ministerio de Economía y Finanzas (MEF) canaliza un presupuesto para el Sector Educación para la "Inclusión de niños, niñas y jóvenes con discapacidad en la Educación Básica y Técnico Productiva; siendo uno de sus productos: № 300573, actividad N 5004303. Que "Personas con discapacidad leve o moderada acceden a instituciones educativas públicas inclusivas con condiciones para su atención" (MED, 2013, p. 209) y, en el que se especifica los bienes y/o servicios que recibirán las instituciones inclusivas (MED, 2013):

Las condiciones adecuadas para su atención implican: » Espacios físicos accesibles: El local escolar debe estar adecuado para facilitar la accesibilidad física (rampa, pasamanos, entre otros), con pisos alineados, baños con apoyos y señalética específicas por discapacidad ( $p$. 112)

Como toda propuesta de mejora requiere una evaluación y seguimiento que permita valorar el éxito de la misma, se vio por conveniente crear un instrumento que nos permita medir el nivel de accesibilidad que presentan las instituciones educativas inclusivas, para lo cual se ha tomado como referente la "Guía orientativa sobre la accesibilidad en los centros educativos" (Aragall, 2010)

El Ministerio de Educación Español, de acuerdo con el Comité Español de Representantes de Personas con Discapacidad (CERMI), constituyó el Foro para la Atención Educativa a Personas con Discapacidad. En dicho foro se propuso diseñar una guía orientativa sobre la accesibilidad en los 
centros educativos, cuyo propósito fue realizar recomendaciones para favorecer a las personas con discapacidad la accesibilidad a los espacios educativos y al currículo.

Fue a partir del análisis de éste documento que elaboramos un instrumento, que nos permitió conocer las adaptaciones de acceso que han realizado o están en proceso de ejecución en las Instituciones Educativas de la RED 02 (Ex-RED19).

\section{Método}

Se realizó un estudio de tipo descriptivo, instrumental dado que se encaminó al desarrollo y análisis psicométrico de un instrumento (León y Montero, 2002) en una muestra de 14 Instituciones Educativas Inclusivas y sus respectivos directivos (13 directores y 01 subdirectora) de la RED 02 , en la cual se recogió información sobre las adaptaciones de acceso que se han hecho, o están en vías de realizarse para hacer de las instituciones espacios educativos accesibles a todas y cada una de las personas.

Se evaluó la presencia, ejecución en proceso o ausencia de adaptaciones de acceso, utilizándose la técnica psicométrica y como instrumento El "Cuestionario sobre adaptaciones de acceso para directores de las Instituciones Educativas Inclusivas", el cual fue elaborado por las autoras del artículo Bernaola y Quintana (2016), quienes desarrollaron esta escala multidimensional teniendo como base la "Guía orientativa sobre la accesibilidad en los centros educativos" creada por La Comisión de Atención Educativa del Foro para la Atención Educativa (Aragall, 2010), cuyo propósito fue realizar recomendaciones para favorecer a las personas con discapacidad la accesibilidad a los espacios educativos y al currículo.

Se crearon ítems 28 que fueron sometidos a juicio de 7 expertos en la materia. Una vez obtenida la validez de contenido se aplicó a la muestra elegida, es decir directivos, que voluntariamente aceptaron desarrollar la prueba, cuyos datos permitieron hacer el análisis psicométrico del instrumento de modo que se cuente con un instrumento útil y con las suficientes garantías psicométricas.

De los tres aspectos que desarrolla la guía (Espacio, Recursos Materiales y Currículo sólo nos abocamos al de Espacio, el cual abarca tres ámbitos: Contexto local, Centro escolar y aula. El análisis factorial de la Escala se llevó a cabo teniendo en cuenta los tres ámbitos:

Factor 1: Contexto local: entorno físico, transporte, excursiones y visitas culturales. Abarca aquellos aspectos relacionados con la utilización de las instalaciones, el acercamiento y la posibilidad de llegar a la I.E. La existencia y señalización de estacionamientos para personas con silla de ruedas, velocidad mínima de circulación escrita en las pistas cercanas a la escuela, señalización acústica y luminosa para avisar el inicio del horario escolar de plazas de aparcamiento accesibles, puertas de acceso principal fácilmente localizable, puertas transparentes cuentan con señalización horizontal que contrasta con el entorno, intercomunicador y timbre de la puerta sencillos de manipular, contar con información anticipada de comunicación y espacio en instalaciones fuera del centro (excursiones, salidas y visitas) para garantizar el acceso. Este factor 
comprendió al inicio los ítems del 1 al 7, los que luego de se hallara el índice de homogeneidad, quedaron solo 4 ítems $(1,2,3$, y 5), siendo retirados los ítems 4,6 y 7.

Factor 2: Centro escolar: comedor, bibliotecas, laboratorios, talleres: este componente incluye: contar con rampas, pasamanos, organización del patio, presencia de paneles informativos para visualizar los mensajes emitidos por megáfono, baños y lavamanos adaptados, puertas de aulas y servicios con manija y pestillo fácil de utilizar 'por personas con dificultades motoras, con prótesis y férulas, suelo antideslizante, ancho de los pasillos, alarmas sonoros y luminosos, juegos seguros y diseñados, carteles de identificación de las diferentes salas (letras y dibujos). Este factor contenía inicialmente 13 ítems del cuestionario: del 8 al 20, pero luego de obtenido el índice de homogeneidad se retiraron 4 ítems (11, 12, 18 y 20), quedando sólo 9.

Factor 3: Contexto Aula: Incluye aspectos relacionados con ancho de las puertas, espacio libre detrás de las mismas, color de las puertas, ventanas, armarios empotrados, papeleras, etc.; acústica de las aulas, acceso adecuado a la carpeta, iluminación uniforme y apropiada, ubicación de la pizarra; contar con paneles o carteles colocados a una altura que permite su lectura. El cuestionario contenía al principio en éste ámbito 8 ítems: del 21 al 28, de los cuales luego del análisis del índice de homogeneidad se retiraron 3 ítems $(21,22$, y 27), quedando 5 (23, 24, 25 , 26, 28).

\section{Resultados}

A continuación se muestran los resultados del análisis de los ítems y de los relacionados a la estimación de la fiabilidad y validez del "CUESTIONARIO SOBRE ADAPTACIONES DE ACCESO PARA LOS DIRECTORES DE LAS INSTITUCIONES DE LAS INSTITUCIONES EDUCATIVAS INCLUSIVAS", además se presentan los resultados de un análisis descriptivo de las respuestas brindadas al cuestionario por los sujetos participantes del estudio.

\section{Descripción y análisis}

Antes de iniciar la presentación de los resultados, se caracterizará a la muestra estudiada.

\begin{tabular}{|l|c|c|c|c|}
\hline & Género & Frecuencia & Porcentaje & Porcentaje válido \\
\hline Válidos & Mujer & 11 & 78,6 & 78,6 \\
\hline & Varón & 3 & 21,4 & 21,4 \\
\hline & Total & 14 & 100,0 & 100,0 \\
\hline
\end{tabular}

Figura1: Distribución de la muestra de género

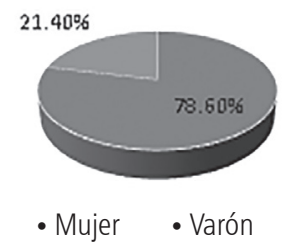


La muestra estuvo conformada por catorce directores de las instituciones educativas de la Ex Red 10 de la Molina. Se halló la distribución de la muestra según Género. En cuanto al género, de los 14 directores participantes el $78.6 \%$ eran mujeres y el $21.4 \%$ eran varones, tal como se observa en la Tabla 1 y Figura

1.1. Análisis de los ítems. La calidad individual de los ítems fue estimada mediante el Índice de Homogeneidad $(\mathrm{IH})$ que se obtuvo calculando el coeficiente de correlación de Pearson cuyo valor se corrigió.

"Eliminando el efecto del propio ítem sobre el puntaje total (correlación ítem-total corregido o ítem remainder); se fijó como criterio de aceptabilidad del ítem el valor 0,20 como mínimo (Ary, Jacobs y Razavieh, 1999). El IH informa el grado de semejanza, de relación entre las respuestas a un determinado ítem y el resto de los ítems de la escala. Es decir, informa el grado en que dicho ítem está midiendo lo mismo que la escala global" (Aliaga, Ponce, Bulnes, Elizalde, Montgomery, Gutiérrez, Delgado, Perea, Torchiani, 2012:174).

Tabla 2: Medias, desviaciones estándares, índice de homogeneidad (IH) de los ítems de la Escala Contexto Local

\begin{tabular}{|c|c|c|c|}
\hline $\mathbf{N}^{\circ}$ Item & Media & Desviación Est. & IH \\
\hline 1 & 1,43 & 0,852 &, 486 \\
\hline 2 & 1,21 &, 426 &, 451 \\
\hline 3 & 1,50 &, 760 &, 499 \\
\hline 4 & 2,57 &, 756 &, 128 \\
\hline 5 & 1,29 &, 726 &, 220 \\
\hline 6 & 2,29 &, 914 &,- 227 \\
\hline 7 & 2,29 &, 914 &, 141 \\
\hline
\end{tabular}

En la Tabla 2 se observa que los ítems 4, 6 y 7 tienen índices de homogeneidad (IH) inferiores a 0,20, lo que psicométricamente significa que estos ítems no miden lo mismo que el resto de ítems componentes de la escala Contexto Local (Entorno) por lo que fueron separados de la escala y se procedió a hacer el re-cálculo del IH sin estos ítems.

Tabla 3: Medias, desviaciones estándares, índice de homogeneidad (IH) de los ítems de la Escala Contexto Local

\begin{tabular}{|c|c|c|c|}
\hline $\mathbf{N}^{\circ}$ Ítem & Media & Desviación Est. & IH \\
\hline 1 & 1,43 & 0,852 &, 492 \\
\hline 2 & 1,21 &, 426 &, 436 \\
\hline 3 & 1,50 &, 760 &, 492 \\
\hline 5 & 1,29 &, 726 &, 504 \\
\hline
\end{tabular}


Se observa en esta Tabla 3 que todos los IH son superiores a 0,20, indicando que los ítems miden lo mismo que mide la escala.

Tabla 4: Medias, desviaciones estándares, índice de homogeneidad (IH) de los ítems de la Escala Centro escolar

\begin{tabular}{|c|c|c|c|}
\hline $\mathbf{N}^{\circ}$ Ítem & Media & Desviación Est. & IH \\
\hline 8 & 1,71 &, 825 &, 252 \\
\hline 9 & 2,14 &, 864 &, 251 \\
\hline 10 & 1,71 &, 994 &, 200 \\
\hline 11 & 2,86 &, 363 &, 125 \\
\hline 12 & 1,57 &, 852 &, 565 \\
\hline 13 & 1,50 &, 855 &, 260 \\
\hline 14 & 1,57 &, 756 &, 434 \\
\hline 15 & 2,29 &, 914 &, 658 \\
\hline 16 & 2,21 &, 893 &, 540 \\
\hline 17 & 1,93 &, 997 &, 158 \\
\hline 18 & 1,86 &, 949 &, 108 \\
\hline 19 & 2,07 &, 997 &, 961 \\
\hline 20 & 2,00 & & \\
\hline
\end{tabular}

En la Tabla 4 se observa que los ítems 11, 12, 18 y 20 tienen índices de homogeneidad (IH) inferiores a 0,20, estos ítems no miden lo mismo que mide la escala Centro escolar, por lo que fueron eliminados y se procedió a hacer el re-cálculo del IH sin estos ítems.

Tabla 5: Medias, desviaciones estándares, índice de homogeneidad (IH) de los ítems de la Escala Centro escolar

\begin{tabular}{|c|c|c|c|}
\hline $\mathbf{N}^{\circ}$ Ítem & Media & Desviación Est. & IH \\
\hline 8 & 1,71 &, 825 &, 383 \\
\hline 9 & 2,14 &, 864 &, 285 \\
\hline 10 & 1,71 &, 994 &, 360 \\
\hline 13 & 1,50 &, 855 &, 625 \\
\hline 14 & 1,57 &, 756 &, 385 \\
\hline 15 & 2,29 &, 914 &, 366 \\
\hline 16 & 2,21 &, 893 &, 499 \\
\hline 17 & 1,93 &, 997 &, 470 \\
\hline 19 & 2,07 &, 997 &, 485 \\
\hline
\end{tabular}

Se observa en esta Tabla 5 que todos los IH son superiores a 0,20, indicando que los ítems miden lo mismo que mide la escala Centro Escolar. 
Tabla 6: Medias, desviaciones estándares, índice de homogeneidad (IH) de los ítems de la Escala Contexto aula

\begin{tabular}{|c|c|c|c|}
\hline $\mathbf{N}^{\circ}$ Ítem & Media & Desviación Est. & IH \\
\hline 21 & 2,86 &, 535 &,- 264 \\
\hline 22 & 2,86 &, 363 &,- 041 \\
\hline 23 & 2,00 &, 961 &, 357 \\
\hline 24 & 2,79 &, 579 &, 445 \\
\hline 25 & 2,29 &, 994 &, 812 \\
\hline 26 & 2,71 &, 726 &, 576 \\
\hline 27 & 2,93 &, 267 &, 194 \\
\hline 28 & 2,64 &, 745 &, 501 \\
\hline
\end{tabular}

En la Tabla 6 se observa que los ítems 21, 22, y 27 tienen índices de homogeneidad (IH) inferiores a 0,20 , estos ítems no miden lo mismo que mide la escala Contexto aula, por lo que fueron eliminados y se procedió a hacer el re-cálculo del IH sin estos ítems.

Tabla 7: Medias, desviaciones estándares, índice de homogeneidad (IH) de los ítems de la Escala Contexto aula

\begin{tabular}{|c|c|c|c|}
\hline $\mathbf{N}^{\circ}$ Ítem & Media & Desviación Est. & IH \\
\hline 23 & 2,00 &, 961 &, 465 \\
\hline 24 & 2,79 &, 579 &, 498 \\
\hline 25 & 2,29 &, 994 &, 773 \\
\hline 26 & 2,71 &, 726 &, 639 \\
\hline 28 & 2,64 &, 745 &, 484 \\
\hline
\end{tabular}

Se observa en esta Tabla 7 que todos los IH son superiores a 0,20, indicando que los ítems miden lo mismo que mide la escala Contexto aula.

1.2. Fiabilidad. El establecimiento de la fiabilidad tiene por objetivo estimar el error existente en la medida, su indicador es el coeficiente de confiabilidad (Aliaga, Ponce, Bernaola, Pecho, 2000: 17). El cálculo de este coeficiente se realizó utilizando el método de la consistencia interna, entendida como intercorrelación entre los ítems utilizando el coeficiente alfa de Crombach.

Tabla 8. Estadísticos descriptivos y coeficiente alfa de Crombach del Cuestionario sobre adaptaciones de acceso para Directores de las IEI

\begin{tabular}{|l|c|c|c|c|}
\hline & $\begin{array}{c}\text { Escala } \\
\text { Contexto local }\end{array}$ & $\begin{array}{c}\text { Escala Centro } \\
\text { escolar }\end{array}$ & $\begin{array}{c}\text { Escala } \\
\text { Contexto aula }\end{array}$ & Escala Total \\
\hline Media & 5,43 & 19,00 & 12,43 & 35,00 \\
\hline Desviación estándar & 2,02 & 4,91 & 2,98 & 5,18 \\
\hline$N^{\circ}$ de ítems & 4 & 9 & 5 & 18 \\
\hline Alfa de Crombach & 0,695 & 0,734 & 0,787 & 0,701 \\
\hline
\end{tabular}


En la Tabla 8 se observa la consistencia interna de las puntuaciones en las escalas del cuestionario. Los coeficientes alfa varían desde 0,695 hasta 0,787. Existen varias propuestas acerca de los valores mínimos del coeficiente alfa. Por ejemplo, Aliaga et al (2012: 181) citan a George y Mallery (1995) quienes "presentan una gama de intervalos de valores de alfa para evaluar la fiabilidad: por debajo de 0,50, fiabilidad inaceptable; entre 0,50 y 0,60, fiabilidad pobre; entre 0,60 y 0,70, fiabilidad de nivel débil; entre 0,70 y 0,80, fiabilidad aceptable; entre 0,80 y 0,90 fiabilidad de un nivel bueno; un valor superior a 0,90 , fiabilidad excelente". Al comparar los coeficientes obtenidos en la muestra de estudio con estos valores, se encuentra que las escalas del Cuestionario sobre adaptaciones de acceso para Directores de las IEI tienen una fiabilidad aceptable, en otros términos un error de medida aceptable.

1.3 Validez. La validez es la característica principal de un instrumento de medición. Este concepto refiere a al grado en que el instrumento está midiendo el constructo o atributo que se supone mide, en este caso las adaptaciones de acceso a las IEI. Las evidencias de la validez proceden de varias fuentes: Evidencias con base en el contenido del instrumento, evidencias basadas en los procesos de respuesta, evidencias de basadas en la relación con otras variables, evidencias basadas en las consecuencias del uso del test, y evidencias con base en la estructura interna del test (AERA, APA, NCME, 2014). En este estudio se enfoca el análisis de la validez del Cuestionario sobre adaptaciones de acceso para Directores de las IEI analizando su estructura interna empleando la técnica de la intercorrelación de las escalas del instrumento. Se espera que las escalas no estén correlacionadas en un grado elevado (superior a 0,80), para sostener que miden aspectos diferenciados del constructo medido.

Tabla 9. Análisis de intercorrelación de las escalas

\begin{tabular}{|l|l|l|l|l|}
\hline & Contexto local & Centro escolar & Contexto aula & \multicolumn{1}{c|}{ Total } \\
\hline Contexto local & ------ & $-0,09$ & $-0,10$ & 0,162 \\
\hline Centro escolar & ------- & & 0,06 & $0,737^{* *}$ \\
\hline Contexto aula & -------- & & & $0,577^{*}$ \\
\hline Total & & & & $-\cdots----$ \\
\hline
\end{tabular}

${ }^{* *}=$ valor estadísticamente muy significativo; ${ }^{*}=$ valor estadísticamente significativo.

En la Tabla 9 se observa que las tres escalas (contexto local, centro escolar y contexto aula) no están correlacionadas entre ellas indicando que miden aspectos específicos independientes entre sí, por otro lado, la escala centro escolar covaría en un nivel muy significativo con la puntuación total del cuestionario, y la escala contexto aula correlaciona con el mismo total en un nivel estadísticamente significativo. En otros términos, la escala Centro escolar representa a la puntuación total, haciéndolo en segundo lugar la escala Contexto aula.

Por tanto, empíricamente las escalas contexto local, centro escolar y contexto aula miden aspectos bastante independientes entre sí, encontrándose reflejada en la puntuación total con mayor peso Centro escolar y Contexto aula, y en menor peso Contexto local. 
En suma, la apreciación de la información del análisis de los ítems, de los coeficientes alfa y de la intercorrelación de las escalas del Cuestionario de acceso a las IEI, permite decir que en la muestra analizada el cuestionario tiene un comportamiento psicométrico de características básicas que lo hace útil para cumplir con el propósito para el que fue construido.

\subsection{Análisis porcentual de las respuestas}

Tabla 10: Frecuencias y Porcentajes de las respuestas a los ítems de la Escala Contexto Local

\begin{tabular}{|c|c|c|c|c|}
\hline $\mathbf{N}^{\circ}$ Ítem & $\mathbf{S i}$ & En proceso & No & Total \\
\hline 1 & $3(21,4 \%)$ & 0 & $11(78,6 \%$ & $14(100 \%)$ \\
\hline 2 & 0 & $3(21,4 \%)$ & $11(78,6 \%)$ & $14(100 \%)$ \\
\hline 3 & $2(14,3 \%)$ & $3(21,4 \%)$ & $9(64,3$ & $14(100 \%)$ \\
\hline 5 & $2(14,3 \%)$ & 0 & $12(85,7 \%)$ & $14(100 \%)$ \\
\hline
\end{tabular}

Como se puede observar en la tabla 10, la mayoría de los ítems han sido respondidos de forma negativa en más del 75\% de la muestra en lo que respecta al Contexto Local, es decir que aún se mantienen el proceso de ejecutar las adaptaciones de accesibilidad al contexto local, siendo los aspectos que requieren implementar y adaptar, el estacionamiento y su señalización, la velocidad mínima de circulación no está escrita visiblemente en las pistas cercanas al colegio, contar con señalización acústica o luminosa que avise inicio del horario escolar.

Tabla 11: Frecuencias y Porcentajes de las respuestas a los ítems de la Escala Centro escolar

\begin{tabular}{|c|c|c|c|c|}
\hline $\mathbf{N}^{\circ}$ Ítem & $\mathbf{S i}$ & En proceso & No & Total \\
\hline 8 & $3(21,4)$ & $4(28.6)$ & $7(50.0 \%)$ & $14(100 \%)$ \\
\hline 9 & $6(42,9)$ & $4(28,6)$ & $4(28,6)$ & $14(100 \%)$ \\
\hline 10 & $5(35,7)$ & $0(0,0)$ & $9(64,3)$ & $14(100 \%)$ \\
\hline 13 & $3(21,4)$ & $1(7,1)$ & $10(71.4)$ & $14(100 \%)$ \\
\hline 14 & $2(14,3)$ & $4(28,6)$ & $8(57,1)$ & $14(100 \%)$ \\
\hline 15 & $8(57,1)$ & $2(14,3)$ & $4(28,6)$ & $14(100 \%)$ \\
\hline 16 & $7(50,0)$ & $3(21,4)$ & $4(28,6)$ & $14(100 \%)$ \\
\hline 17 & $6(42,9)$ & $1(7,1)$ & $7(50,0)$ & $14(100 \%)$ \\
\hline 19 & $7(50,0)$ & $1(7,1)$ & $6(42,9)$ & $14(100 \%)$ \\
\hline
\end{tabular}

En cuanto al Factor Centro Escolar, los resultados son dispares, en algunos aspectos se observa un avance en las adaptaciones, tanto si se ejecutaron o están en proceso, y en otros aspectos aún no se han considerado su ejecución en casi un $50 \%$ o más del $50 \%$ de la muestra. 
Tabla 12: Frecuencias y Porcentajes de las respuestas a los ítems de la Escala Contexto aula

\begin{tabular}{|c|c|c|c|c|}
\hline $\mathbf{N}^{\circ}$ Ítem & $\mathbf{S i}$ & En proceso & No & Total \\
\hline 23 & $6(42,9)$ & $2(14,3)$ & $6(42,9)$ & $14(100 \%)$ \\
\hline 24 & $12(85,7)$ & $1(7,1)$ & $1(7,1)$ & $14(100 \%)$ \\
\hline 25 & $9(64,3)$ & $0(0,0)$ & $5(35,7)$ & $14(100 \%)$ \\
\hline 26 & $12(85,7)$ & $0(0,0)$ & $2(14,3)$ & $14(100 \%)$ \\
\hline 28 & $11(78,6)$ & $1(7,1)$ & $2(14,3)$ & $14(100 \%)$ \\
\hline
\end{tabular}

En la tabla 12, se puede percibir que el Factor Contexto Aula, es el que ha obtenido en la mayoría de los ítems más del 60\% respuestas positivas, lo que indica que los directivos y docentes de las escuelas inclusivas han logrado con sus propios recursos las adecuaciones en las aulas para atender a las necesidades de sus estudiantes.

\section{Conclusiones}

- La calidad individual de los ítems fue estimada mediante el Índice de Homogeneidad (IH), hallándose 10 ítems cuyos índices de homogeneidad (IH) fueron inferiores a 0,20, 10 que psicométricamente significa que estos ítems no miden lo mismo que el resto de ítems componentes de la escala, por lo que fueron retirados.

- El análisis de consistencia interna de las puntuaciones en las escalas del cuestionario muestran que los coeficientes alfa varían desde 0,695 hasta 0,787 , lo que indica que tienen una fiabilidad aceptable.

- En cuanto a su validez el análisis de su estructura interna empleando la técnica de la intercorrelación de las escalas del instrumento arrojo que las tres escalas (contexto local, centro escolar y contexto aula) no están correlacionadas entre ellas indicando que miden aspectos específicos independientes entre sí, por otro lado, la escala centro escolar covaría en un nivel muy significativo con la puntuación total del cuestionario, y la escala contexto aula correlaciona con el mismo total en un nivel estadísticamente significativo.

- La Escala que obtuvo predominantemente un porcentaje alto de respuestas positivas fue Contexto Aula, lo que implica que los directivos han expresado que sus instituciones educativas han incluido en sus aulas el mayor número de adaptaciones de acceso, y posiblemente con el apoyo de sus recursos propios para poder atender a las necesidades acceso y educativas de sus estudiantes inclusivos.

- Las escalas en las que se obtuvieron predominantemente un porcentaje menor de respuestas positivas fueron las dimensiones: Contexto Local y Centro Escolar. Estos resultados nos muestran que aún están en proceso las acciones para que las instituciones sean accesibles todos y cada uno de las personas

- La apreciación de la información del análisis de los ítems, de los coeficientes alfa y de la intercorrelación de las escalas del Cuestionario de acceso a las IEI, permite decir que en la muestra analizada el cuestionario tiene un comportamiento psicométrico de características básicas que lo hace útil para cumplir con el propósito para el que fue construido. 


\section{CUESTIONARIO SOBRE ADAPTACIONES DE ACCESO PARA DIRECTORES DE LAS IEI}

Estimado (a) director (a), el presente cuestionario tiene como objetivo recoger información sobre las adaptaciones de acceso que se han hecho, o están en vías de realizarse para hacer de las instituciones espacios educativos accesibles a todas y cada una de las personas.

Por favor, lea con detenimiento cada una de las afirmaciones y marque con un aspa según sea su contexto:

Sí (Si ha llevado a cabo o cuenta con los accesos).

En proceso (Si está llevando a cabo los trámites o su ejecución).

No (Cuando no se cuenta con las adaptaciones de acceso).

\begin{tabular}{|c|c|c|c|c|}
\hline No & ADAPTACIONES DE ACCESO & \multirow[t]{2}{*}{ Sí } & \multirow{2}{*}{$\begin{array}{c}\text { En } \\
\text { proceso }\end{array}$} & \multirow[t]{2}{*}{ No } \\
\hline I & $\begin{array}{c}\text { Contexto local: entorno físico, transporte, excursiones y } \\
\text { visitas culturales. }\end{array}$ & & & \\
\hline 1 & $\begin{array}{l}\text { Existe y está señalizado un estacionamiento para personas con silla de ruedas } \\
\text { y, éste está cerca de los accesos peatonales y de las entradas accesibles a la } \\
\text { institución educativa. }\end{array}$ & & & \\
\hline 2 & $\begin{array}{l}\text { La velocidad mínima de circulación en las cercanías del colegio está escrita } \\
\text { visiblemente en las pistas. }\end{array}$ & & & \\
\hline 3 & $\begin{array}{l}\text { La escuela cuenta con una señalización acústica que avisa el inicio del horario } \\
\text { escolar, para llamar la atención de las personas que van distraídas y, luminosa } \\
\text { para que la perciban las personas con limitaciones auditivas. }\end{array}$ & & & \\
\hline 4 & $\begin{array}{l}\text { Las puertas con hojas totalmente transparentes disponen de señalización } \\
\text { horizontal que contraste con el entorno, para hacerlo perceptible a las personas } \\
\text { con discapacidad visual. }\end{array}$ & & & \\
\hline II & $\begin{array}{c}\text { Centro escolar: comedor, bibliotecas, laboratorios, } \\
\text { talleres }\end{array}$ & & & \\
\hline 5 & $\begin{array}{l}\text { La institución educativa cuenta con rampas para acceder en silla de ruedas a } \\
\text { las aulas, para los alumnos que llevan mochilas con ruedas o para transportar } \\
\text { material (de limpieza, proyectores) de un lugar a otro. }\end{array}$ & & & \\
\hline 6 & $\begin{array}{l}\text { Los pequeños desniveles (uno o dos escalones) cuentan con rampas adaptadas, } \\
\text { que cubren la totalidad del ancho del escalón. }\end{array}$ & & & \\
\hline 07 & $\begin{array}{l}\text { Existen pasamanos en ambos lados de las rampas, a lo largo de todo su } \\
\text { recorrido y, en caso de que algún usuario del centro lo precise, están instalados } \\
\text { también en los pasillos. }\end{array}$ & & & \\
\hline 08 & $\begin{array}{l}\text { Los servicios higiénicos cuentan con un baño y lavamanos con suficiente } \\
\text { espacio para ser utilizado por personas con silla de ruedas. }\end{array}$ & & & \\
\hline
\end{tabular}




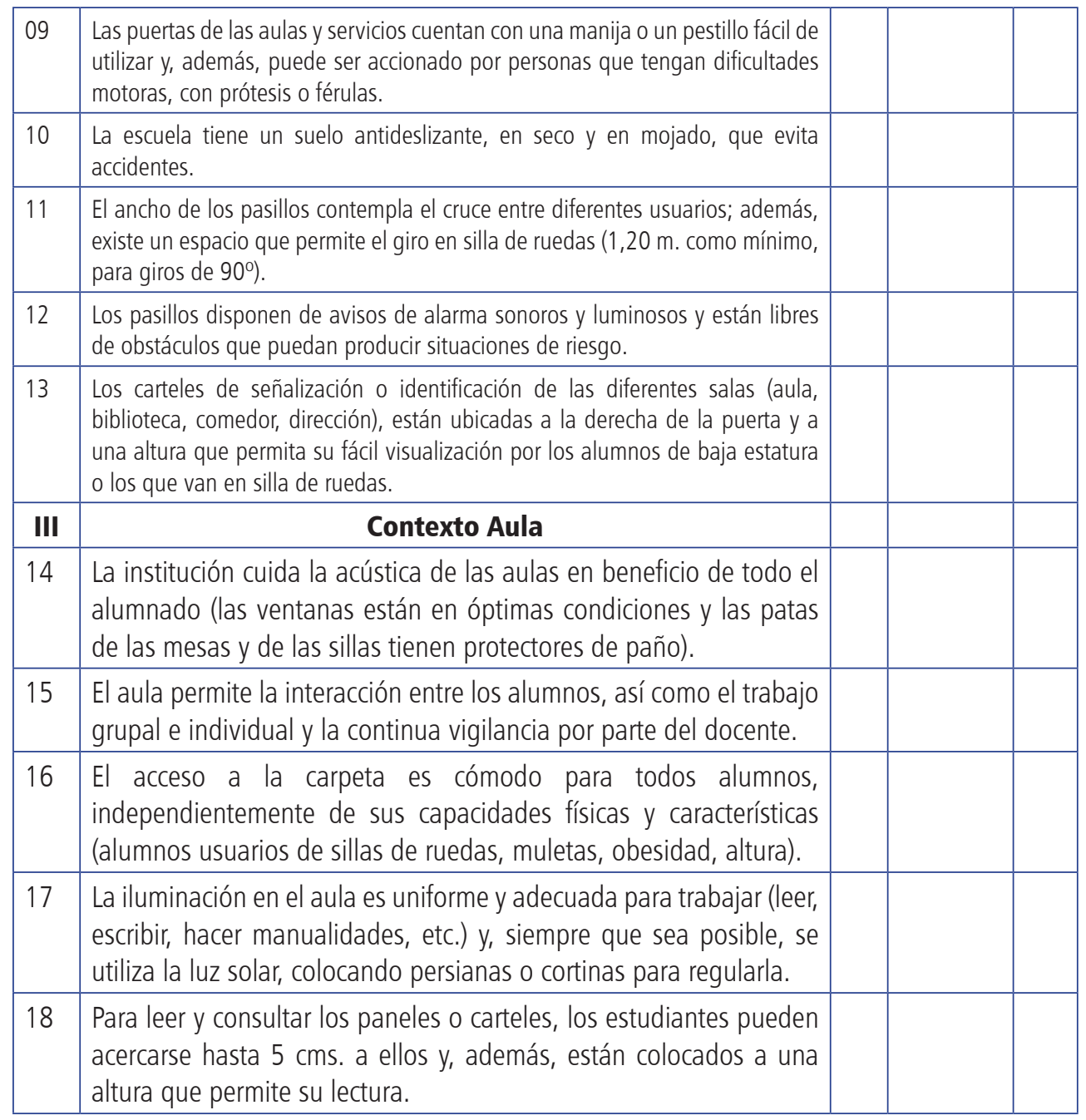




\section{REFERENCIAS}

Aragall, F. (2010). La accesibilidad en los centros educativos. Madrid, España: Ediciones Cinca, Telefónica y CERMI

Naciones Unidas (2009) Convención sobre los derechos de las personas con discapacidad. Enable. Recuperado en http://www.un.org/spanish/disabilities/default.asp?id=497

Ministerio de Educación (2013) Programa presupuestal 0106. Inclusión de niños, niñas y jóvenes con discapacidad en la educación básica y técnico productiva. Recuperado en https:// mef.gob.pe/contenidos/presu_publ/ppr/prog_presupuestal/articulados/0106_NNA_ discapacidad_educacion_productiva.pdf

Aliaga T. J., Ponce, D. C., Bulnes B. M., Elizalde B. R., Montgomery U. W., Gutiérrez O. V., Torchiani, G. R. (2012). Las inteligencias múltiples: evaluación y relación con el rendimiento en matemática en estudiantes del quinto año de secundaria de Lima Metropolitana. Revista de Investigación en Psicología, 15(2), 163-202.

Aliaga, T. L., Ponce, C., Bernaola, E., Pecho, J. (2001). Características Psicométricas del Inventario de Autoevaluación de la Ansiedad ante Exámenes (IDASE). Paradigmas. Colegio de Psicólogos del Perú. Consejo Directivo Regional I., Lima, Año 2, Vol, 2, Nos. 3 y 4. 11-30. 